\title{
Avaliação do bem-estar animal por meio da caracterização e quantificação de lesões em carcaças de bovinos abatidos em frigorífico exportador no estado de Goiás, Brasil
}

\author{
[Evaluation of animal welfare through the characterization and quantification of injuries in \\ slaughtered cattle in exporting refrigerators in the state of Goiás, Brazil]
}

\section{"Artigo Científico/Scientific Article"}

\author{
Rian Carvalho Silva, Layra Soares Lessa de Pré, João Nélson dos Santos Morais Neto, Thaís \\ Sampaio Ornellas, Suzane Vitória Freitas Morais, Francimar Fernandes Gomes*
}

\begin{abstract}
Laboratório de Morfologia e Patologia Animal (LMPA), Centro de Ciências e Tecnologias Agropecuárias (CCTA), Universidade Estadual do Norte Fluminense Darcy Ribeiro (UENF), Campos dos Goytacazes- RJ, Brasil.

*Autor para correspondência/Corresponding author: E-mail. fernandes_rj@hotmail.com
\end{abstract}

\begin{abstract}
Resumo
O presente trabalho teve como objetivos caracterizar e quantificar as lesões de bovinos enviados para abate em um frigorífico localizado na região centro-oeste do estado de Goiás, Brasil, cuja carne é destinada principalmente à exportação. As lesões foram classificadas de acordo com sua extensão e gravidade em: grau 1 - hematoma leve, grau 2 - hematoma intermediário e grau 3 - fratura. A ocorrência de tais lesões foi relacionada a condutas inadequadas de manejo pré-abate no âmbito do estabelecimento sendo para tanto considerados: densidade animal nos currais de espera, deslizamentos e quedas no desembarque, uso de bastão elétrico desde o desembarque até o box de insensibilização e número de vocalizações durante a condução dos animais. Ao final, foi observado que $100 \%$ dos animais apresentaram algum tipo de lesão sendo que as mais frequentes foram as de grau $1(100 \%)$ seguidas de $2 \%$ de frequência para as lesões de grau 2. Estas foram relacionadas a falhas ocorridas fora do abatedouro frigorífico nas etapas de embarque e transporte tendo em vista que o estabelecimento atendeu os requisitos de boas práticas sob o ponto de vista do bem-estar animal.
\end{abstract}

Palavras-chave: manejo; inspeção; estresse; comportamento; bovino de corte.

\begin{abstract}
The present study characterized and quantified lesions in bovines slaughtered in a slaughterhouse in the Midwestern region of the state of Goiás, Brazil. Most of the meat obtained is exported. The lesions were scored according to size and severity as grade 1 (mild hematoma), grade 2 (intermediate hematoma), and grade 3 (fracture). The occurrence of these lesions was evaluated considering in-house pre-slaughter management. The variables analyzed were the number of animals in waiting corrals, slips and falls during unloading, use of stunning wand during unloading through the stunning box, and number of vocalizations during handling of animals. The results show that $100 \%$ of animals presented some kind of lesion, mostly grade $1(100 \%$ of animals), followed by grade $2(2 \%)$. These lesions were linked with problems outside the slaughterhouse during loading and transportation, in view of the fact that the slaughterhouse met good practices requirements in animal welfare.
\end{abstract}

Keywords: beef cattle; behavior; inspection; management; stress.

\section{Introdução}

Os primeiros princípios sobre bem-estar animal começaram a ser estudados no ano de 1965 por um comitê formado por pesquisadores e profissionais relacionados à agricultura e pecuária do Reino Unido da Grã-Bretanha, denominado Comitê Brambell, iniciando-se, assim, um estudo mais aprofundado sobre seus conceitos e 
definições. Esse Comitê constituiu uma resposta à pressão da população, indignada com os maus tratos dados aos animais em sistemas de confinamento, denunciados no livro "Animal Machines" (Animais Máquinas), publicado pela jornalista inglesa Ruth Harrison em 1964.

Atualmente pode se dizer que bem-estar significa qualidade de vida e, para sua mensuração, o Comitê Brambell desenvolveu o conceito das "Cinco Liberdades", que foram aprimoradas pelo Farm Animal Welfare Council - FAWC (Conselho de bem-estar na Produção Animal) do Reino Unido e têm sido adotadas mundialmente. As Cinco Liberdades são: 1) livre de fome, sede e má nutrição; 2) livre de desconforto; 3) livre de dor, injúria e doença; 4) livre para expressar seu comportamento normal e 5) livre de medo e diestresse (Ludtke et al., 2012).

No que tange à legislação brasileira, é importante salientar o disposto no artigo 88 do Regulamento de Inspeção Industrial e Sanitária de Origem Animal - RIISPOA (BRASIL, 2020). Este determina que a administração do estabelecimento fica obrigada a tomar as medidas no sentido de serem evitados maus tratos aos animais, pelos quais é responsável desde o momento do seu desembarque.

$\mathrm{O}$ artigo 103 do mesmo regulamento cita que é proibido o abate de animais que não tenham permanecido em descanso, jejum e dieta hídrica, respeitadas as particularidades de cada espécie e as situações emergenciais que comprometem o bemestar animal, além de desobedecer ou não observar os preceitos de bem-estar animal referentes aos produtos de origem animal.

Na prática, o que vem sendo observado é que as contusões são indicativas de manejo inadequado em qualquer etapa do processo de abate, durante o transporte, desembarque e no frigorífico. As principais causas de contusões são decorrentes da movimentação muito rápida dos animais, pisos molhados, escorregadios e irregulares, projeções pontiagudas em portões, paredes, períodos longos de dieta e estresse crônico (Civeira et al., 2006).

O manejo pré-abate envolve uma série de situações que podem causar estresse nos animais como, por exemplo, o uso abusivo de choque elétrico nas propriedades rurais, determinando o aumento na frequência de hematomas na carcaça (Grandin, 2000).

No que concerne ao transporte, Andrade et al. (2008) afirmaram que o mesmo representa uma importante causa de lesões em animais, sobretudo quando submetidos à série sucessiva de manejo e transportados em estradas não-pavimentadas por longas distâncias. Por sua vez, Civeira et al. (2006) relataram a densidade de cargas muito baixas ou elevadas durante essa etapa como causa de contusões.

Outras situações que contribuem para a ocorrência de lesões são as curvas e freadas em alta velocidade. No desembarque, a tentativa de vários animais saírem ao mesmo tempo também é determinante para a ocorrência de impactos nas regiões posterior e lateral (Foz, 1999).

\section{Material e Métodos}

O trabalho foi realizado em um abatedouro frigorífico sob fiscalização do Serviço de Inspeção Federal, localizado no estado de Goiás, Brasil. Este possui capacidade para abater 2400 animais por dia, sendo voltado para o mercado interno e externo, exportando para países como a Suécia, Irã, Finlândia, Rússia, China e Chile. Foram avaliados lotes de bovinos com número mínimo de cinco e máximo de 50 animais, perfazendo o total de 1386 carcaças, sendo estas oriundas de fêmeas e machos em quantidades não padronizadas e analisadas de forma aleatória.

O manejo pré-abate foi avaliado de acordo com o percentual de atendimento aos parâmetros: densidade, número de vocalizações, uso do bastão elétrico, bem como o número de deslizamentos e quedas.

Em cada etapa da movimentação foram examinados por volta de 100 animais de acordo com os critérios de auditoria em bem-estar descritos por Ludtke et al. (2012) sendo considerados os seguintes padrões de conformidade:

I) Densidade nos currais: $2,5 \mathrm{~m}^{2} / \mathrm{animal}$.

II) Deslizamentos e quedas:

a) Excelente: sem escorregões ou quedas.

b) Aceitável: escorregão sem tocar o chão.

c) Não aceitável: tocar o chão.

III) Uso do Bastão elétrico:

a) Aceitável $=13$ a $25 \%$.

b) Não aceitável = 26 a $49 \%$.

c) Sérios problemas = Acima de $49 \%$.

IV) Número de vocalizações:

a) Excelente: até $0,5 \%$ dos bovinos vocalizam.

b) Aceitável: 3\% dos bovinos vocalizam.

c) Inaceitável: 4 a 10\% vocalizam.

d) Problema sério: mais de $10 \%$ vocalizam. 
Após a esfola de cada animal, foi feita a classificação das lesões de acordo com sua extensão e gravidade (Figura 1) conforme o descrito na literatura especializada (Ludtke et al., 2012). Foram considerados hematomas as lesões traumáticas com ruptura de vasos sanguíneos, com acúmulo de sangue e soro, sem descontinuidade cutânea (Nanni Costa et al., 2006).

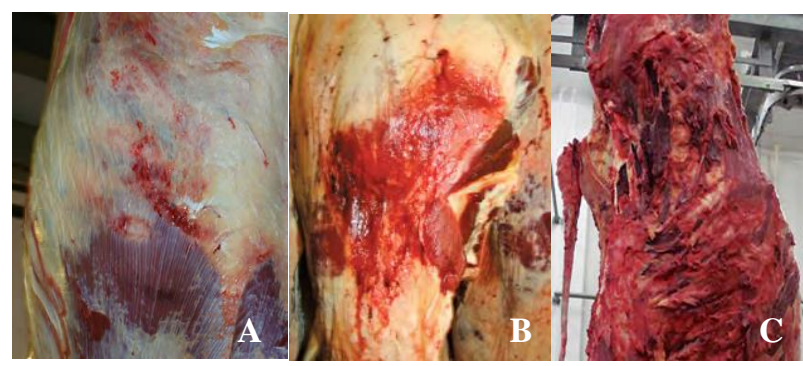

Figura 1. Tipos de lesões comumente observadas em carcaças de animais enviados para abate. A) Grau 1 - hematoma leve que afeta apenas o tecido subcutâneo. B) Grau 2 - hematoma intermediário que afeta o tecido subcutâneo e muscular. C) Grau 3 - Fratura que afeta os tecidos subcutâneo, muscular e ósseo. Fonte: Ludtke et al. (2012).

Os dados foram analisados em planilha do software Microsoft Excel mediante a determinação do percentual de cada tipo de lesão e a gravidade destas foi associada ao sexo da espécie avaliada, bem como, a más condutas de manejo observadas no período pré-abate.

\section{Resultados e discussão}

Verificou-se após a análise de 1386 carcaças (1297 machos e 89 fêmeas), que todas possuíam algum tipo de lesão, sendo a maioria correspondente a pequenos hematomas de grau 1, localizados majoritariamente nos membros pélvicos (Figura 2A).

No que tange ao percentual de lesões, resultados similares foram observados por Jarvis et al. (1995) que, em pesquisa realizada na Austrália, também constataram elevada frequência (97\%). Tal fato foi corroborado por Andrade et al. (2008) que, no Pantanal Sul-Mato-Grossense, também encontraram uma ou mais lesões em $84,3 \%$ das carcaças.

Todos os autores citados afirmaram que o manejo pré-abate inadequado compromete o bemestar animal e a qualidade das carcaças. As lesões são causadas por fatores diversos que podem ocorrer durante as últimas 24 horas de vida dos animais, assim sendo, o manejo dos animais durante o período ante mortem, pode ter um efeito significativo na redução da qualidade da carcaça.

Contrariando os resultados obtidos por Jarvis et al. (1995), Nascimento et al. (2009), em trabalho desenvolvido no estado do Pará, Brasil, observaram a presença de contusões em menor frequência; $66 \%$ dos animais avaliados. Tal disparidade pode ser atribuída a diferenças nas condições das estradas e rodovias ou nos cuidados adotados pelos motoristas durante o transporte, somado as curvas acentuadas e passagem por regiões onde a manutenção da pista é precária. Outro fator é a distância entre os estabelecimentos de abate e as propriedades de origem que pode contribuir para a disparidade no número e na extensão de lesões em cada local de estudo.

Um fato que chamou atenção foi a diferença quanto ao número de hematomas de grau 2, observado entre machos e fêmeas. Tendo-se para esse tipo de lesão a frequência de $100 \%$ em fêmeas e nenhuma em machos. Isso pode ser explicado pela menor camada de musculatura e menor resistência física das vacas em comparação aos machos. Estas tendem a se deitar mais durante o transporte, sendo então pisoteadas. Para os machos foram observadas apenas lesões de grau 1 (100\%).

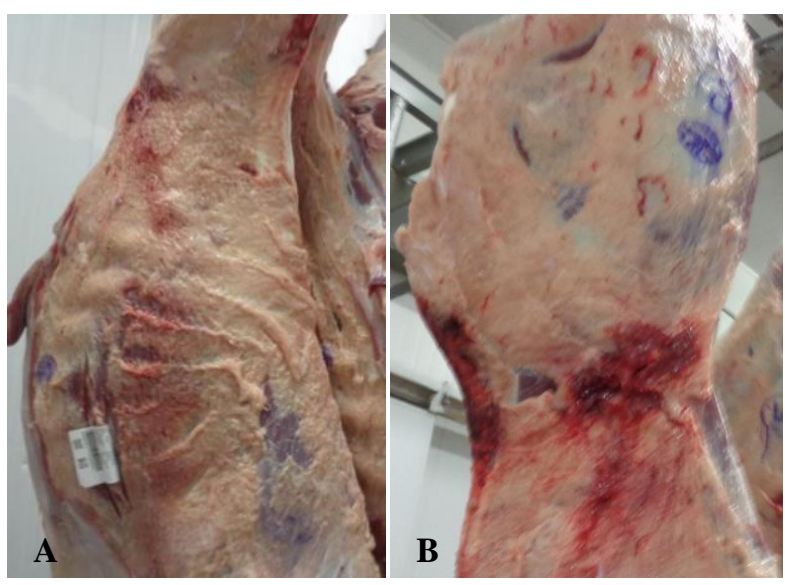

Figura 2. Caracterização de lesões em carcaças de bovinos abatidos em frigorífico exportador. A) Grau 1 - hematoma leve que afeta apenas o tecido subcutâneo. B) Grau 2 - hematoma intermediário que afeta o tecido subcutâneo e muscular.

Diferentemente do que foi observado na presente pesquisa, Civeira et al. (2006) apontaram um número expressivo de machos e fêmeas que possuíam contusões em grau 2. Nesse caso, tal discrepância pode ser explicada por fatores como a ausência/mau treinamento dos funcionários e precariedade nas condições de tráfego nas estradas. E em relação à extensão das lesões verificou-se que nos quartos traseiros, as contusões superficiais 
(grau 1) foram mais prevalentes nos machos $(65,1 \%)$, enquanto nas fêmeas, $50,8 \%$ das contusões foram de grau 2.

Apesar das lesões graves terem ocorrido em menor proporção em comparação às lesões brandas (Tabela 1), as mesmas ainda poderiam ser minimizadas mediante a intensificação do treinamento de funcionários em bem-estar, visto que, alguns manejadores foram flagrados fazendo uso de bastões elétricos inadequadamente a uma frequência de $16 \%$ mesmo quando os animais já estavam em movimento, contrariando o preconizado por autores como Ludtke et al. (2012).

Tabela 1. Frequência de lesões de acordo com o grau observado em bovinos abatidos em frigorífico no estado de Goiás.

\begin{tabular}{|c|c|c|c|c|c|c|}
\hline \multirow{2}{*}{ Tipos de lesões } & \multicolumn{2}{|c|}{ Machos } & \multicolumn{2}{|c|}{ Fêmeas } & \multicolumn{2}{|c|}{ Total } \\
\hline & (n) & $(\%)$ & (n) & $(\%)$ & $(n=1386)$ & $(\%)$ \\
\hline Grau 1 & 1297 & 93,6 & 89 & 6,4 & 1386 & 100 \\
\hline Grau 2 & - & - & 28 & 2 & 28 & 2 \\
\hline Grau 3 & - & - & - & - & - & - \\
\hline
\end{tabular}

Ainda no que concerne ao uso inadequado do bastão elétrico, este também ocorreu durante a etapa de desembarque quando o manejador não esperava tempo suficiente para que os animais se familiarizassem com o novo ambiente. Os funcionários alegavam a necessidade de apressar o desembarque para buscar a próxima "carga". De acordo com Sá et al. (2020) o bastão elétrico costuma ser utilizado de forma rotineira e abusiva. Tal constatação foi feita após avaliarem o manejo pré-abate de 500 bovídeos em três abatedouros no estado do Amazonas, Brasil, tendo observado para dois estabelecimentos que na maioria dos animais, o bastão era aplicado por mais de dois segundos. Ademais, em diversas situações faltava espaço para movimentação, e, por conseguinte, os indivíduos vocalizavam, deslizavam ou caíam por conta desta prática.

Sobre a densidade animal nos currais de espera, observou-se $100 \%$ de conformidade em relação ao conceito de bem-estar, visto que os animais não evidenciavam sinais de desconforto, pois expressavam seu comportamento natural na companhia de membros do mesmo lote de acordo com suas necessidades sociais, respeitando-se o conceito das cinco liberdades preconizado pela Farm Animal Welfare Council (2009).

Nesta pesquisa, um parâmetro para o qual o resultado da avaliação foi considerado um sério problema se refere ao número de escorregões (Tabela 2).

Segundo Ludtke et al. (2012), considera-se escorregão a situação em que após o deslizamento, $o$ ventre do animal não encosta no chão. Portanto, o elevado percentual de escorregões observado no presente trabalho sugere a necessidade de mudanças na estrutura do piso das rampas de modo a dificultar a derrapagem. Isso porque o excesso de sujidades aliado à elevada frequência de lavagem predispunha os animais a um excessivo número de deslizamentos (Figura 3). É importante enfatizar que a angulação das rampas observada nessa pesquisa atendia aos parâmetros de bem-estar descritos na literatura, sendo no máximo 20 graus, o que permite aos animais não escorregar numa frequência equivalente a $3 \%$ das vezes.

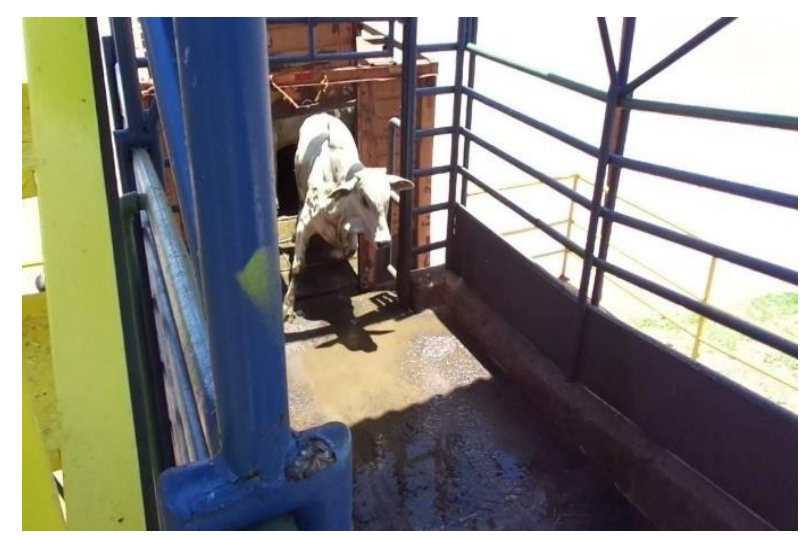

Figura 3. Registro do deslizamento de um bovino no momento da chegada ao abatedouro frigorífico devido à presença de umidade e sujidades na rampa de desembarque que predispunha os animais a elevada frequência de contusões.

Apesar do elevado número de escorregões observado, surpreendentemente verificou-se nessa pesquisa, resultado satisfatório quanto ao número de quedas (Tabela 2). Esses resultados estão de acordo com os reportados por Ludtke et al. (2010), de forma que os requisitos de bem-estar preconizados foram atendidos.

Outro dado importante é o número de vocalizações. Em pesquisa realizada por Bertoloni et al. (2012), o autor obteve o percentual de 4,3\% sendo, portanto, não aceitável. O mesmo também afirmou que o transporte feito em caminhões de deck simples reduz o percentual para $1,47 \%$. Na presente pesquisa observou-se que a vocalização 
atingiu 3\%, estando, portanto, dentro do limite aceitável sob o ponto de vista do bem-estar.

Também é relevante discutir o fato de muitas lesões detectadas nos abatedouros frigoríficos não se originarem a partir de más condutas dos profissionais que trabalham nesse tipo de estabelecimento e, sim, a partir de maus tratos ocorridos no âmbito das propriedades rurais. Petroni et al. (2013), afirmaram que produtores não capacitados quanto ao tema usualmente cometem falhas nas etapas de embarque e transporte. Salientaram, também, que a ausência de um programa de bem-estar animal capaz de interligar o início e o final da cadeia produtiva de carnes acarreta desprestígio no mercado internacional e enormes perdas devido à remoção de partes das carcaças acometidas por lesões.

Em geral, pode-se afirmar que no estabelecimento monitorado o conceito de bemestar animal foi atendido, visto que, dentre os cinco parâmetros de avaliação considerados na Tabela 2, quatro apresentaram frequências compatíveis com práticas desejáveis de manejo pré-abate. Em contrapartida, para o percentual de escorregões, a frequência observada denotou a necessidade de adoção de medidas corretivas direcionadas em caráter emergencial.

Tabela 2. Resultado da avaliação do bem-estar no manejo pré-abate de bovinos em abatedouro frigorífico exportador: porcentagem de casos observados nas etapas de desembarque, espera e movimentação dos animais até o box de insensibilização.

\begin{tabular}{lcccc}
\hline Parâmetro/ local & Excelente & Aceitável & Não aceitável & Problema sério \\
\hline Densidade ${ }^{\text {I }}$ (currais de espera: $\left.\mathrm{n}=24\right)$ & $100 \%$ & - & - & - \\
Escorregões ${ }^{\mathrm{II}}$ (desembarque: $\left.\mathrm{n}=160\right)$ & - & - & - & $88 \%$ \\
Quedas II (desembarque: $\mathrm{n}=160)$ & - & $2,5 \%$ & - & - \\
Uso do bastão elétrico $^{\mathrm{III}}$ (seringa: $\left.\mathrm{n}=200\right)$ & - & $16 \%$ & - & - \\
Vocalização $^{\text {IV }}$ (do brete a seringa: $\left.\mathrm{n}=200\right)$ & - & $3 \%$ & - & - \\
\hline
\end{tabular}

I Densidade adequada: $2,5 \mathrm{~m}^{2} / \mathrm{animal}$; Excelente $=76$ a $100 \%$ dos currais com densidade adequada; Aceitável $=51$ a $75 \%$ dos currais; Não aceitável $=26$ a 50\% de superlotação; Problema sério $=$ todos os currais com elevada densidade. II Deslizamentos ou quedas: Excelente $=$ sem deslizamento ou quedas, Aceitável = deslizamentos em menos de 3\% dos animais; Não aceitável = 1\% de quedas; Problema sério $=5 \%$ de quedas ou mais de $15 \%$ de escorregões. III Uso do Bastão: Aceitável = 13 a 25\%; Não aceitável = 26 a $49 \%$, Sérios problemas = Acima de $49 \%$. IV Vocalização: Excelente $=$ até $0,5 \%$ dos bovinos vocalizam; Aceitável $=$ até $3 \%$ dos bovinos vocalizam; Não aceitável $=4$ a 10\% vocalizam; Problema sério: mais de $10 \%$ vocalizam.

Vale ressaltar que lesões mais graves (em grau 3) não foram observadas nesse trabalho. Provavelmente, devido ao fato dos motoristas e funcionários realizarem cursos de capacitação em bem-estar, tornando os animais menos propensos a esse tipo de lesão. É sabido que em abatedouros frigoríficos exportadores a fiscalização costuma ser mais criteriosa que a realizada em estabelecimentos não exportadores devido as maiores exigências do mercado externo no que concerne ao quesito qualidade.

Tal fato é explicado por Marra et Al. (2017) ao afirmarem que as indústrias registradas no Serviço de Inspeção Federal - SIF, em função de exigências impostas pelo mercado de exportação, possuem instalações, fluxo de produção e sistemas de controle de qualidade rígidos, o que as impedem de ter perdas comerciais, ao passo que em estabelecimentos menores como os abatedouros municipais registrados no Serviço de Inspeção Municipal - SIM, fatores como o volume menor de negociações e a característica do mercado consumidor, tornam evidentes a existência de uma pressão para a diminuição dos custos de produção da indústria, influenciando as características do ambiente laboral dos trabalhadores.

O principal colaborador para o bem-estar animal na cadeia industrial é ter uma equipe treinada, capacitada, comprometida, atenta e cuidadosa no manejo dos animais, com foco para o comprometimento, pois o funcionário pode ter $\mathrm{o}$ treinamento, mas não o comprometimento de colocar em prática.

\section{Conclusão}

O abatedouro frigorífico atendeu aos requisitos de boas práticas sob o ponto de vista do bem-estar animal, tendo em vista que as lesões observadas em sua maioria não foram associadas a más condutas de manejo observadas no interior do estabelecimento. Provavelmente estas ocorreram nas etapas de embarque e transporte.

Todos os animais apresentaram lesões em grau 1 (leve) ao passo que apenas $2 \%$ apresentaram grau 2 (intermediário).

\section{Conflito de Interesse}

Os autores declaram não existir conflito de interesse. 


\section{Comitê de Ética}

O projeto de pesquisa foi aprovado pelo Comitê de Ética da Universidade Estadual do Norte Fluminense Darcy Ribeiro, sob o protocolo $\mathrm{n}^{\circ}$ $383 / 2018$.

\section{Agradecimentos}

À Universidade Estadual do Norte Fluminense Darcy Ribeiro e ao Conselho Nacional de Desenvolvimento Científico e Tecnológico (CNPq).

\section{Referências}

Andrade, E.N.; Silva, R.A.M.S.; Roça, R.O.; Silva, L.A.C.; Gonçalves, H.C.; Pinheiro, R.S.B. Ocorrência de lesões em carcaças de bovinos de corte no Pantanal em função do transporte. Ciência Rural, 38(7): 1991-1996, 2008.

Bertoloni, W.; Silva, J.L.; Abreu, J.S.; Andreolla, D.L. Bem-estar e taxa de hematomas de bovinos transportados em diferentes distâncias e modelos de carroceria no estado do Mato Grosso - Brasil. Revista Brasileira de Saúde e Produção Animal, 13(3): 850-859, 2012.

BRASIL. Ministério da Agricultura, Pecuária e Abastecimento. Instrução Normativa ${ }^{\circ} 56$ de 2008. Disponível em: $<$ https://www.gov.br/agricultura/ptbr/assuntos/sustentabilidade/bem-estaranimal/arquivos/arquivos-legislacao/in-56-de2008.pdf>. Acesso em: 14 jan. 2021.

BRASIL. Ministério da Agricultura, Pecuária e Abastecimento. Decreto $\mathrm{n}^{\circ} \mathbf{1 0 . 4 6 8}$, de 18 de agosto de 2020. Disponível em: <http://www.planalto.gov.br/ccivil_03/_ato201 9-2022/2020/decreto/d10468.htm>. Acesso em: 14 jan. 2021.

Civeira, M.P.; Renner, R.M.; Vargas, R.E.S.; Rodrigues, N.C. Avaliação do bem-estar animal em bovinos abatidos para consumo em frigorífico do Rio Grande do Sul. Veterinária em Foco, 4(1): 5-11, 2006.

Farm Animal Welfare Council. FAWC updates the five freedoms. Veterinary Records, 31: 357, 1992.

Foz, I. Manejo do pré-abate provoca contusão em 50\% dos bovinos. Pecuária de corte, (87): 2732, 1999.

Grandin, T. Introduction management and economic factors of handling and transport. In: . Livestock Handling and Transport. $2^{\text {nd }} \mathrm{ed}$. Wallingford: CABI, 2000. p. 1-14.

Harrison, R. Animal machines: the new factory farming industry. London: Stuart (Vincent) \& J.M. Watkins, 1964. 186p.

Jarvis, A.M.; Selkirk, M.S.; Cockram, M.S. The influence of source, sex class and pre-slaughter handling on the bruising of cattle at two slaughterhouses. Livestock Production Science, 43: 215-224, 1995.

Ludtke, C.B.; Ciocca, J.R.P.; Dandin, T.; Barbalho, P.C.; Vilela, J.A. Abate humanitário de aves. Rio de Janeiro: WSPA, 2010. 120p.

Ludtke, C.B.; Ciocca, J.R.P.; Barbalho, P.C.; Dandin, T.; Vilela, J.A.; Ferrarini, C. Abate humanitário de bovinos. $1^{\mathrm{a}}$ ed. Rio de Janeiro: WSPA, 2012. 148p.

Marra, G.C.; Simone, S.C.; Neto, F.P.B.A.; Cardoso, T.A.O. Avaliação dos riscos ambientais na sala de abate de um matadouro de bovinos. Saúde Debate, 41: 175-187, 2017.

Nanni Costa, L.; Lo Fiego, D.P.; Tassone, F.; Russo, V. The relationship between carcass bruising in bulls and behavior observed during pre-slaughter phases. Veterinary Research Communications, 30: 379-381, 2006.

Nascimento, G.R.; Rodrigues, W.B.; Martins, N.E.X.; Dias, F.E.F.; Cavalcante, T.V.; Freitas, F.L.C.; Leira, M.H.; Almeida, K.S. Avaliação do bem-estar animal em bovinos abatidos no Pará. Revista Veterinária em Foco, 6(2): 121127, 2009.

Peñuela, M.H.R.; Toro, C.G.; Valencia, J.A.S. Evaluación del manejo pre-sacrificio y su relación com la presencia de contusiones em canales bovinas. Biosalud, 10(2): 28-36, 2011.

Petroni, R.; Bürger, K.P.; Gonçalez, P.O.; Rossi, G.A.M.; Vidal-Martins, A.M.C.; Aguilar, C. E.G. Ocorrência de contusões em carcaças bovinas em frigorífico. Revista Brasileira de Saúde e Produção Animal, 14(3): 478-484, 2013.

Sá, A.P.A.; Santos J.F.; Sousa, E.L.; Santo, E.F.E. Avaliação do bem-estar animal no manejo préabate de bovídeos em abatedouros no Amazonas, Brasil. Brazilian Journal of Development, 6(10): 79354-79369, 2020. 